\title{
The computational generative patterns in Indonesian batik
}

\author{
Hokky Situngkir \\ [hs@compsoc.bandungfe.net] \\ Dept. Computational Sociology \\ Bandung Fe Institute
}

March, $7^{\text {th }} 2008$

\begin{abstract}
The paper discusses the terminology behind batik crafting and showed the aspects of self-similarity in its ornaments. Even though a product of batik cannot be reduced merely into its decorative properties, it is shown that computation can capture some interesting aspects in the batik-making ornamentation. There are three methods that can be exploited to the generative batik, i.e.: using fractal as the main source of decorative patterns, the hybrid batik that is emerged from the acquisition of L-System Thue-Morse algorithm for the harmonization within the grand designs by using both fractal images and traditional batik patterns, and using the random image tessellation as well as previous tiling algorithms for generating batik designs. The latest can be delivered by using a broad sources of motifs and traditionally recognized graphics. The paper concludes with certain aspects that shows how the harmony of traditional crafting and modern computation could bring us a more creative aspects of the beautiful harmony inherited in the aesthetic aspects of batik crafting.
\end{abstract}

Keywords: batik, fractal, self-similarity, creativity, aesthetics. 


\section{On what we call Batik?}

Batik has been very famous as an important textile product made by Javanese, Indonesia. There has been a very long historical route of batik as a traditional and cultural heritage in Indonesia (Hasanudin, 2001), even earlier than the era of the first king of Majapahit, Raden Wijaya (1294-1309 A.D.). Despite the popular and common recognition, batik is actually not a monopoly of Javanese culture. Batik has been also recognized as part of traditional textiles Sumatera Island, e.g.: Jambi (Kerlogue, 1997).

In Javanese tradition, from the patterns of decoration and geographical aspects where they are developed, batik textiles can be roughly categorized as Batik Vorstenlanden and Batik Pesisir. Batik Vorstenlanden is the art of textiles that is existed in places like keraton, Javanese traditional government or monarchy, i.e.: Solo and Yogya, while Batik Pesisir is those developed in coastal area, e.g.: Cirebon, INdramayu, Pekalongan,Tuban, Madura (Sondari \& Yusmawati, 2000). As developed, improved, innovated, and crafted in coastal area, the latter are rich with the influence from many culture abroad, e.g.: Chinese, Islamic tradition.

Can we call Batik just by seeing the ornamentation in traditional textiles? The answer is interestingly No, since batik is also realized to be the process of the mbatik. Thus, the batik ornaments cannot be separated from the process of the making. Relating to the previous facts, there exist some differences on crafting Batik respect to the local places and traditions, be it the Batik Vorstenlanden and also different places where Batik Pesisir are produced. Thus, the differences are not only related to the patterns of the ornamentation but also the places where the crafts are designed and produced. Different places and traditions would bring different materials used to colorings, designs, and techniques used. Furthermore, traditional appreciation and usage of batik is not the same to all people for it is somehow directly related to the cosmos of the social system. Different batik crafts are used and wore by different social classes and even occasions; some batik is produced for the statesmen and the other for the ordinary civilians, some are wore for daily events while other are used for particular important occasions. Those are inherent in the cultural heritage where batik is crafted and used and become the source of the complexity of batik.

This fact become the fundamental arguments and simultaneously understanding, that observing batik cannot be reduced to the patterns of decoration, but should be delivered by looking at the textile products as a whole processes from the phases of the production to the aesthetic appreciation. This made batik very interesting and our acquisitions of computation could promise some amusing and unlimited sense of the batik itself as an Indonesian traditional heritage (Doellah, 2002).

However, we could outline that generally speaking, the making processes employ some pseudoalgorithmic steps, namely:

1. Klowongan, the process of the drawing of the basic elements of the general designs.

2. Isen-isen, the process of filing the parts of the drawings finished from the previous step with some motifs. Some patterns regarding to this step, e.g.: cecek, sawut, cecek sawut, sisik melik.

3. Harmonic Ornamentations, the placing of the background of the designs as a whole of the design. Some traditional patterns are employed, like pola ukel, galar, gringsing, or some other variants from the isen, e.g.: sekar sedhah, rembyang, sekar pacar. 


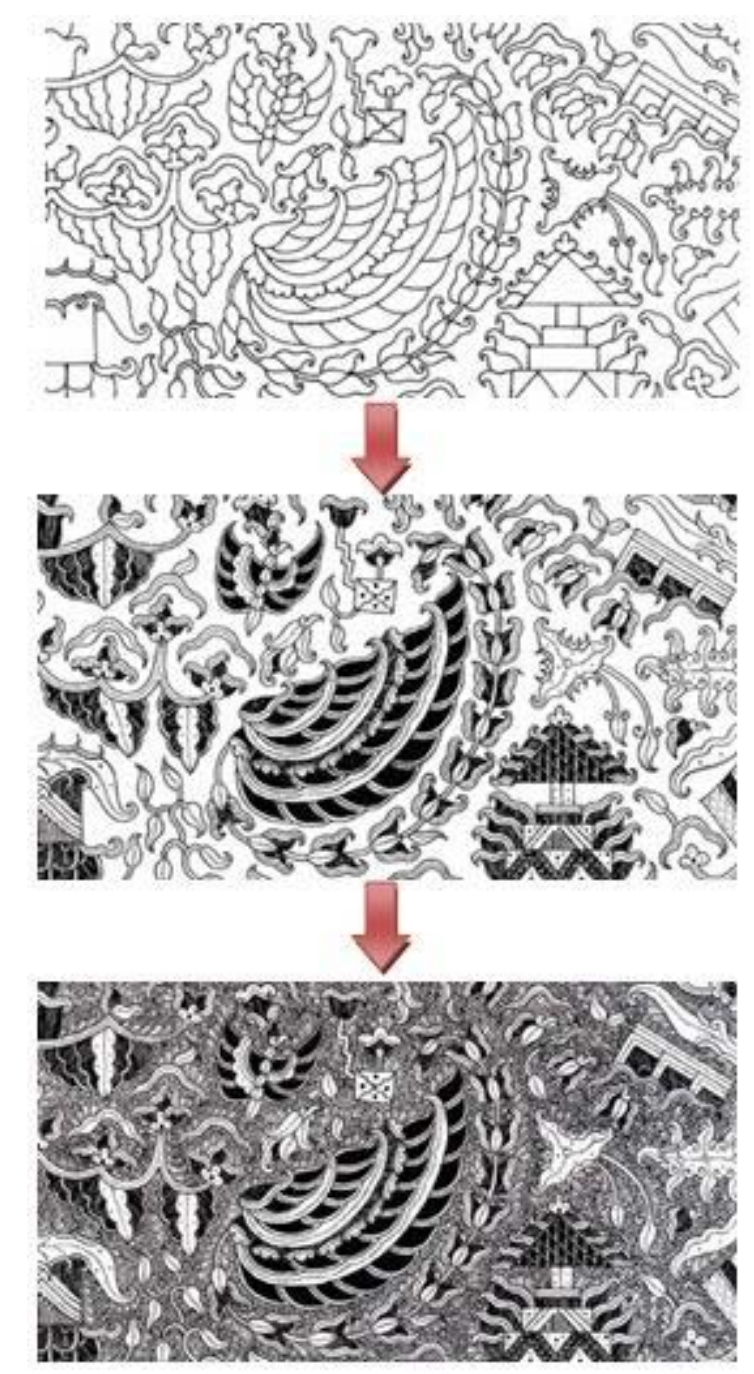

Figure 1. The process of Batik: klowong $\rightarrow$ isen $\rightarrow$ harmonic ornamentations (Doellah, 2002)

Moreover, in its broadest sense, the process of the batik itself cannot be reduced into merely steps of the ornamentations. The materials incorporated in it also play an important role, including the textiles, the organic colorings, the wax, and so on. However, the paper focus on the ornamentation of the batik and how our understanding on fractal geometry and the related computational techniques could wider the creativity and innovation upon batik ornamentation.

\section{Batik and the properties of self-similarity}

The bright Dutch artist, M. C. Escher (1898-1972), was famous with his masterpieces exploiting the geometry of tessellation. Tessellations can be regarded as a (visual) art composition in which patterns made up of one or more shapes, completely covering a surface without any gaps or overlaps (cf. Situngkir, 2005). It is obvious from figure 2 that there exists particular similarity the way we might enjoy the Lunglungan Merak and Escher's wood masterpiece - related to the placement of objects in them. The "harmonious" fillings in the Batik designs, despite apparently much more sophisticated forms relative to Escherian tessellation, is the interesting concept that is emerged.

Self-similarity has played important role in a lot of cultural artifacts as well as the natural ones, from the pigmentation of shells to the architectural designs of megastructures (cf. Wolfram, 2002). Furthermore, the formal system of self-similarity in some particulars aspects can be analytically 
observed in the fractal geometry. This has been in fact becoming an important perspective to see things in al lot of natural phenomena (cf. Bak, 1996) and their mathematical properties (Mandelbrot, 1982). Somehow, the beauty in art and culturally growed aesthetics could also be interestingly discussed in the sense of fractal geometry (cf. Kappraff, 2002 \& Malkevitch, 2003).
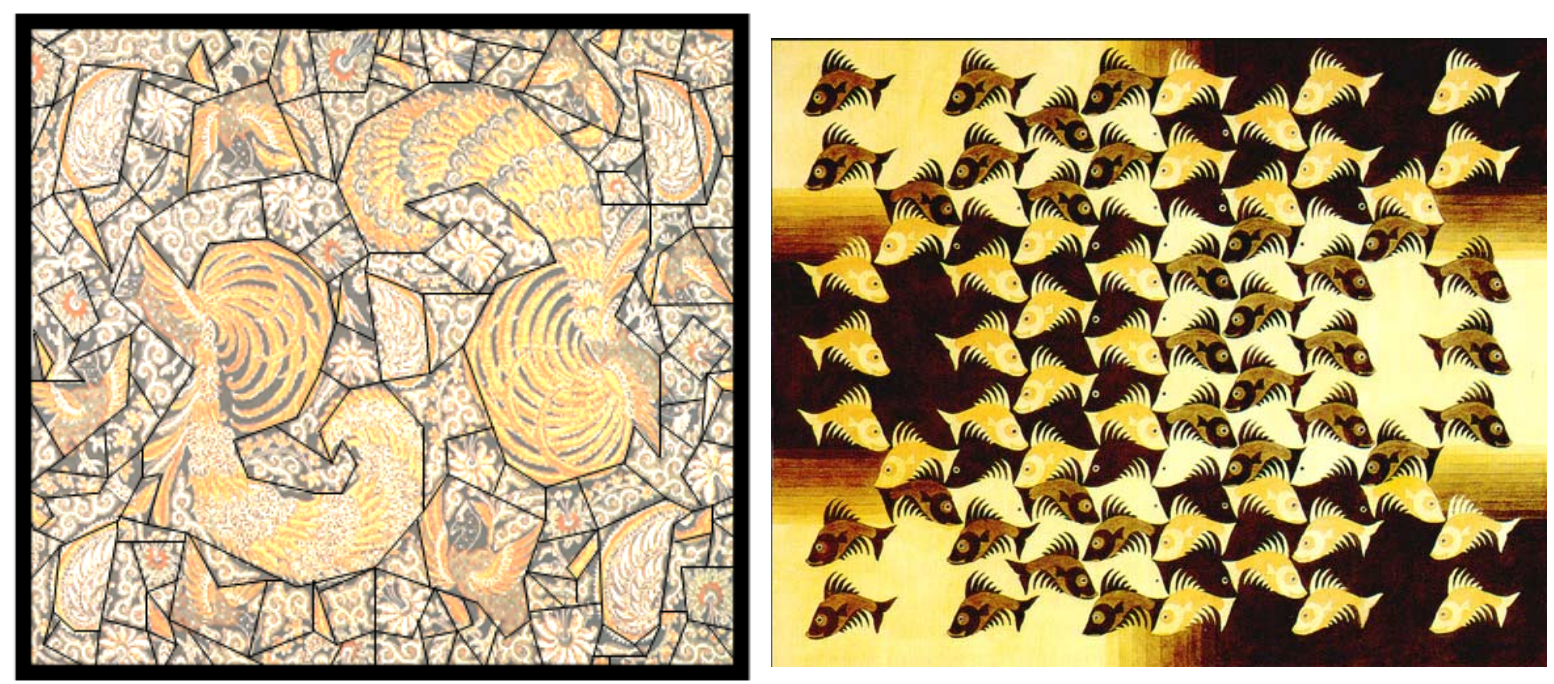

Figure 2.

Left: An illustrative of the reverse-engineering of tessellating batik design form Surakarta knowned as "Lunglungan Merak" (Doellah, 2002) compared to Right: classical Escher's tesselation, the design for wood intarsia panel for Leiden Town Hall (1940).

Thus, what about batik ornaments and their inherent self-similarity? As it has been shown in figure 2 , the reverse engineering (related to the presentation of figure 1) of the batik design could be yielded a sort of Voronoi diagram of the irregular polygons. The rule is simple: painting certain objects by mbatik process to cover all the robe, while the emerging batik is with high complexity. The way a batik-artists painting the robe emerges tessellating patterns in a quite different way Escherian motifs or patterns emerged. This is a very nice way to the appreciation of batik textiles as well as it has been used to enjoy an escherian engraved-wood work of art.

Furthermore, interestingly Hariadi (2008) has tried to show that there could be a conjecture the existence of fractal geometry in batik designs. Furthermore, there is a conjecture that we could see the fractal dimensionality measured in some batik ornaments that could used to classify one another. In the next section, we would like to see that we could also further exploitation to fractal insights and computational iterated algorithms in order to broader the ornamentation techniques of Indonesian batik. The challenge is to have computational acquisition in order to enhance the modern appreciation of the traditional batik ornaments.

\section{Computationally Generated Batik Ornamentation}

Regarding to batik as a whole, a lot of modern products have now wrapped in the motif inspired by batik patterns. However they cannot be called batik whatsoever, for they are not textiles and there is no employment of the mbatik method in its making. Thus we can use a simple formalization of what we can call batik, i.e.:

[A] The process of the making, by using painting devices called canthing, be it cap (stamped batik) or tulis (manually drawn in its detail). This includes the materials used in the processes. 
[B] The decorative ornaments as emerged in the batik textiles.

[C] The appreciation on how to use the batik.

Yet, the set of the properties $[A],[B]$, and $[C]$ present the hierarchy of the thing we call batik in the relation

batik $\equiv C \subset B \subset A$

as showed in figure 3.

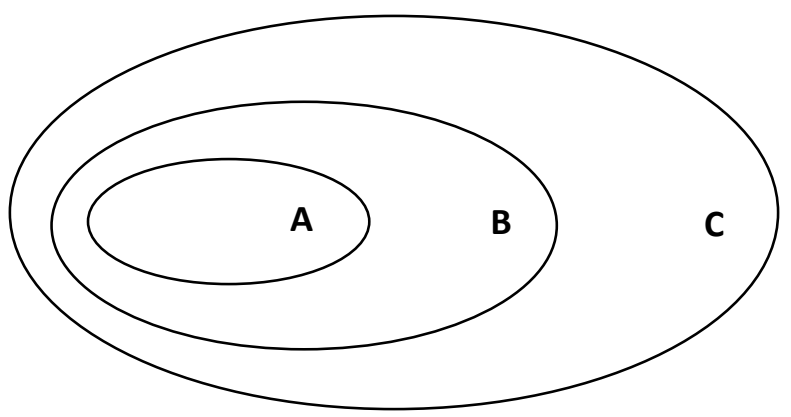

Figure 3.

The relations of the three main properties of batik

Thus, a parang ornament of batik, cannot be called batik as it is attached as the decoration of a wallpaper, for instance, but a caricature of a face, can be called batik if the process of mbatik is used in textiles (set $A$ ) is incorporated in a robe. The patterns of some batik pesisir, including the contemporary ones, are using some ornamenting motifs based on the influence of Arabic/Islamic, Chinese, and even Indian, which in turn do not have to use the steps as shown previously. Still, they are recognized as batik for it is fabricated in the specific processes of mbatik.

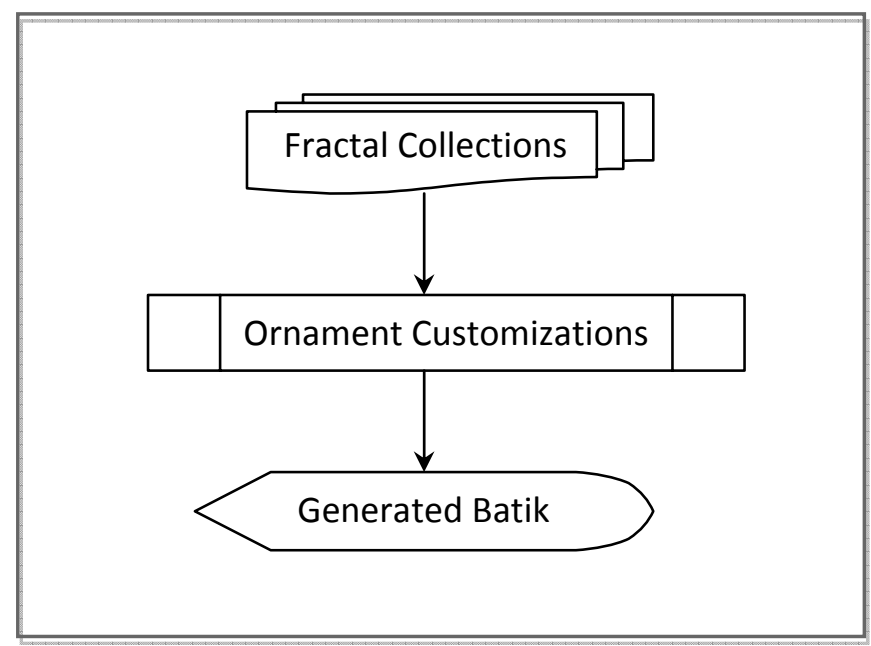

Figure 4.

The Fractal Batik. 
However, the rest of the paper is, in fact, motivated by the formal system of self-similarity patterns in batik in the acquisitions of some interesting mathematical aspects of fractal geometry, the study to the naturally exhibited self-similar systems. Here we introduce some interesting systems of the generations of the emerged complex patterns that can be accorded with batik ornaments: the study of the computationally generated batik decorations.

There are at least four ways on constructing batik ornaments in our acquisitions of the concept of self-similarity and fractals, i.e.:

1) Batik Fractal: Fractals themselves are visually can be designed to be an inspiration of Batik.

The gaze to the artistically colored figure of the Mandelbrot set as well as the Julia set (cf. Mandelbrot, 1982) have always brought to us the beauty of the complexity as emerged from a simple mathematical formulations in complex plane. Both Julia and Mandelbrot set are used as the computational drawing incorporating various available coloring techniques based on the presentation in the complex quadratic polynomials of

$$
F_{c}: z \mapsto z^{2}+c
$$

where $z$ is the complex numbers and $c$ is the complex parameter. The more detail exploration to the sets can be referred to the original text of Mandelbrot (1982) and Peitgen \& Saupe (1988) where interestingly we could see how Julia set can deliberately be recognized as part of the Mandelbrot one. Thus, the two sets are just the sets of complex numbers. However, the sophisticated coloring techniques and the zooming (for the nature of the self-similarities in the emerged figures) on different locations in the complex plane could bring us interesting drawings that can be feed into the inspiration to the batik crafting. Some examples of the figures can be seen in figure 5 and 6 .

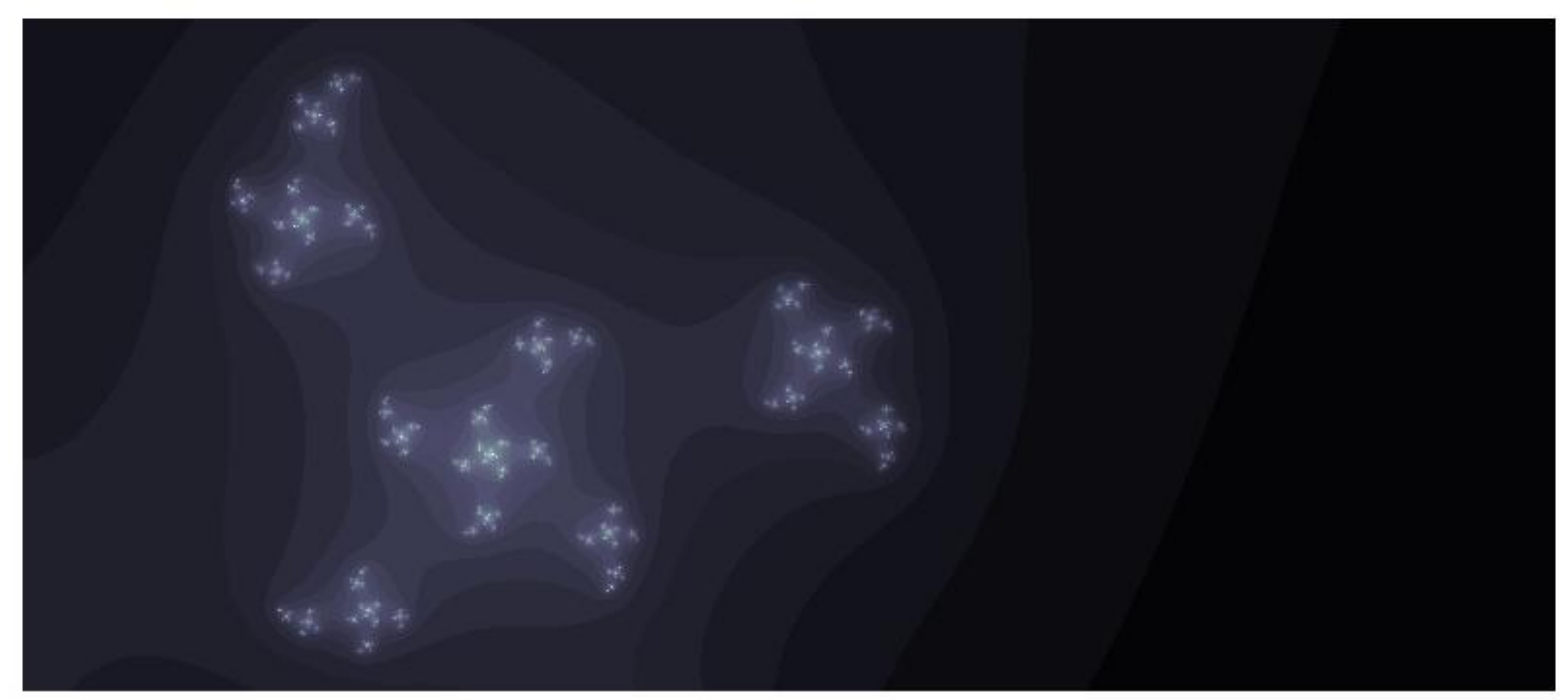

Figure 5.

The Julia sets with the gradation of the corresponding coloring techniques used in the drawing the image (below) 

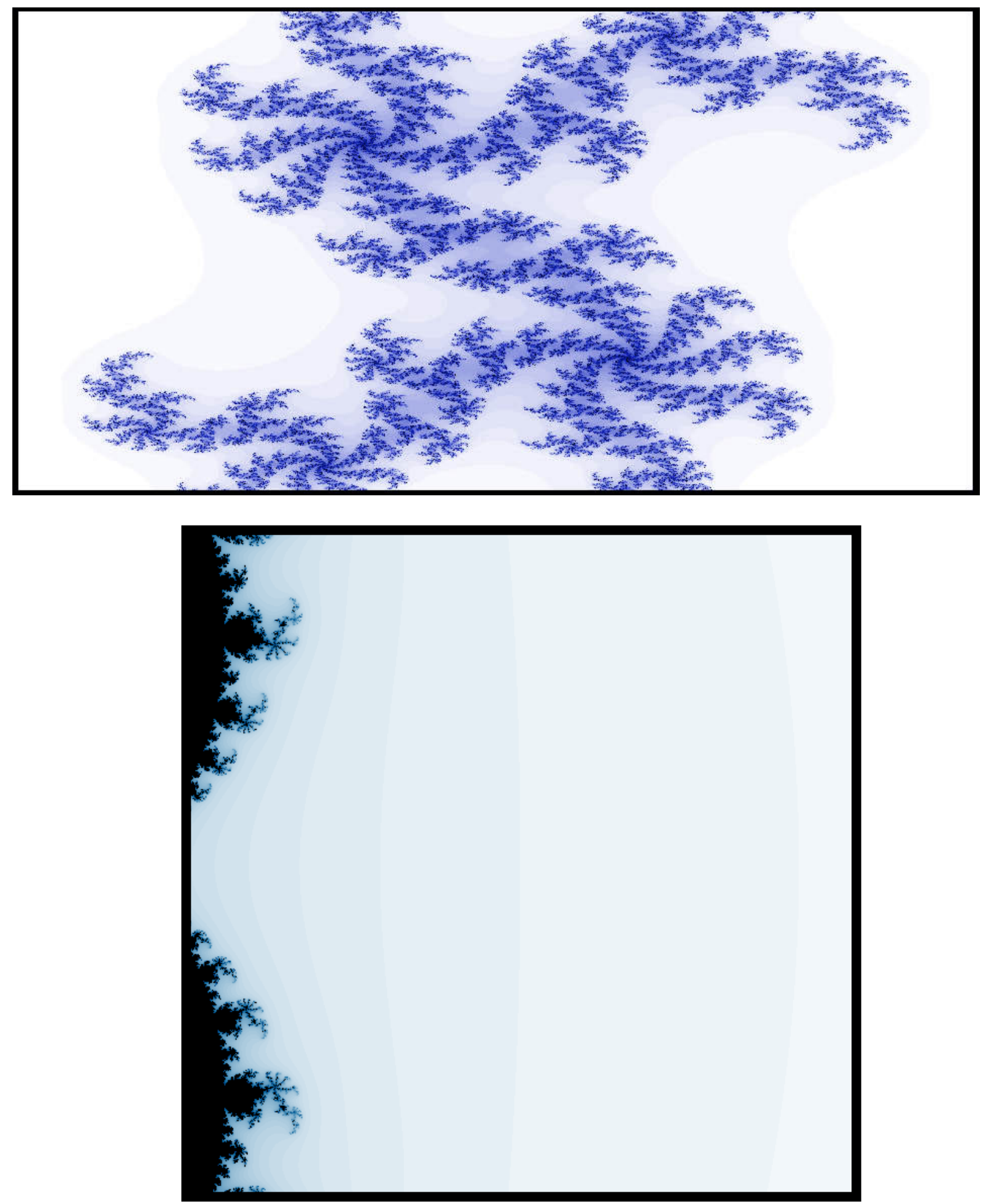

Figure 6.

The Julia and Mandelbrot sets drawings with coloring and interesting different zooming locations and different values of coefficients can be incorporated to the batik crafting processes.

Furthermore, there have been known great deal of fractals and complex geometric shapes that interestingly can be exploited for the broader sense of batik ornamentation, e.g.: the space-fillings, 
Peano curves, Sierpinski triangles, Newton's method, Phoenix set, Koch curves, dragon curve and a lot more as well as their modifications and innovations.

2) Batik Hybrid: The patterns from fractal can be used as the main pattern of the ornaments and decorated with the original isens or basic motif of batik, and vice versa.

As it has been described previously, we can recognize the mbatik process as some kind of pseudoalgorithmic steps with which can be adapted to the computational techniques. The study of fractal geometry has brought us many alternatives on the way of constructing self-similar patterns (Barnsley, 1988). Those ways can be used as a way on how we can computationally enrich the mbatik in its ornamentation.

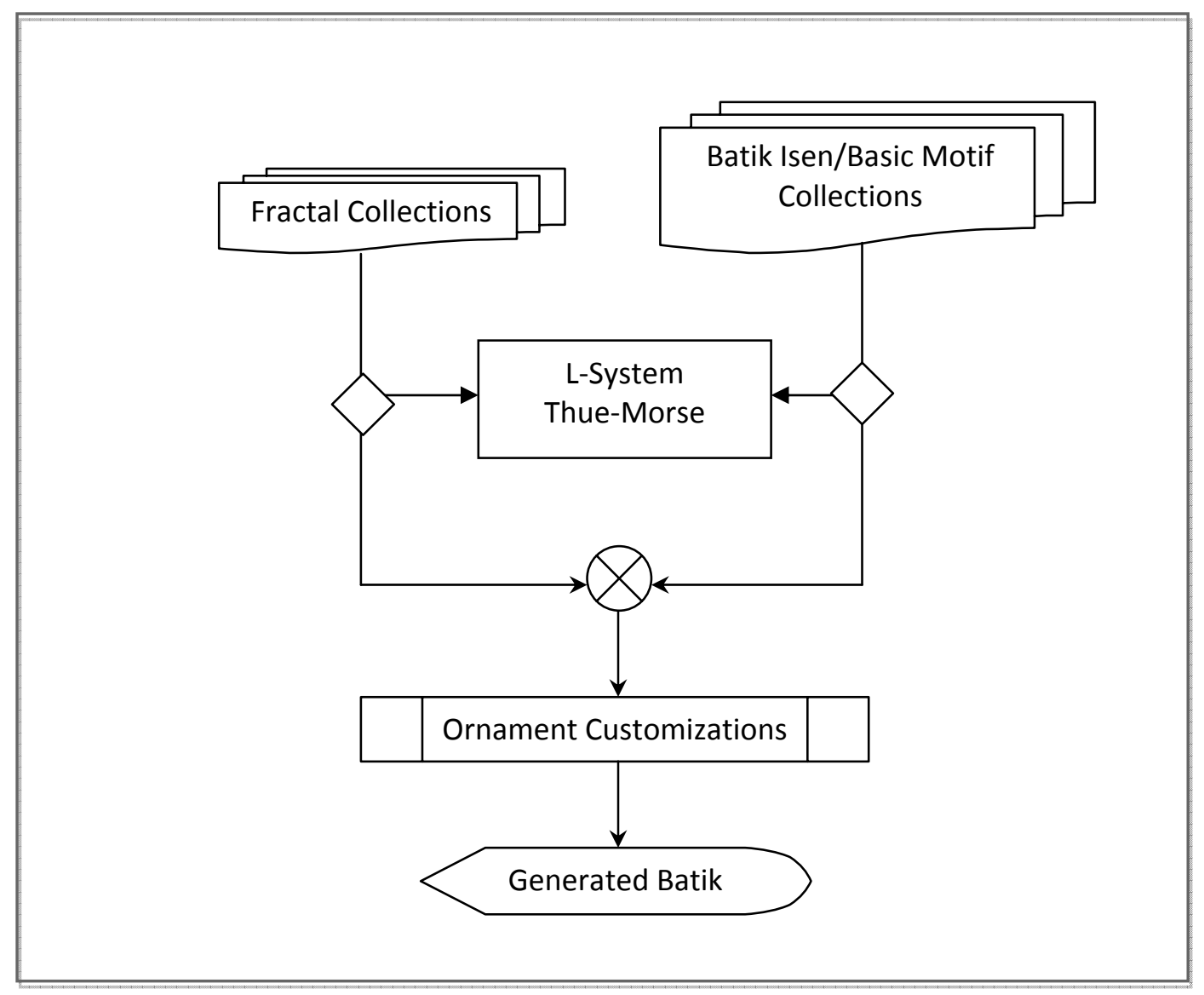

Figure 7.

Batik hybrid generations.

As we know, a method to generate fractals is Iterated Function Systems (IFS) and or L-System. For its space-filling feature it can be exploited to computationally generate isens in batik ornamentation. Examples can be seen in figure 8. The method of tiling using this kind of algorithm as incorporated in the paper is based on the 2-dimensional Thue-Morse algorithm (Wright, 1996). Formally speaking, suppose we have the set of basic elements with two colors represented in binary of $V=\{0,1\}$ and the initial condition is $\omega=0$, we can set the rule as: 
$r_{1}: 0 \rightarrow 1$
$r_{2}: 1 \rightarrow 0$

(3)

or in 1-dimensional we can define it mathematically,

$\prod_{i=0}\left(1-x^{2^{i}}\right)=\sum_{j=0}^{\infty}(-1)^{t_{j}} X^{j}$

where $t_{j}$ is the $j$-th element as we start at $j=0$.

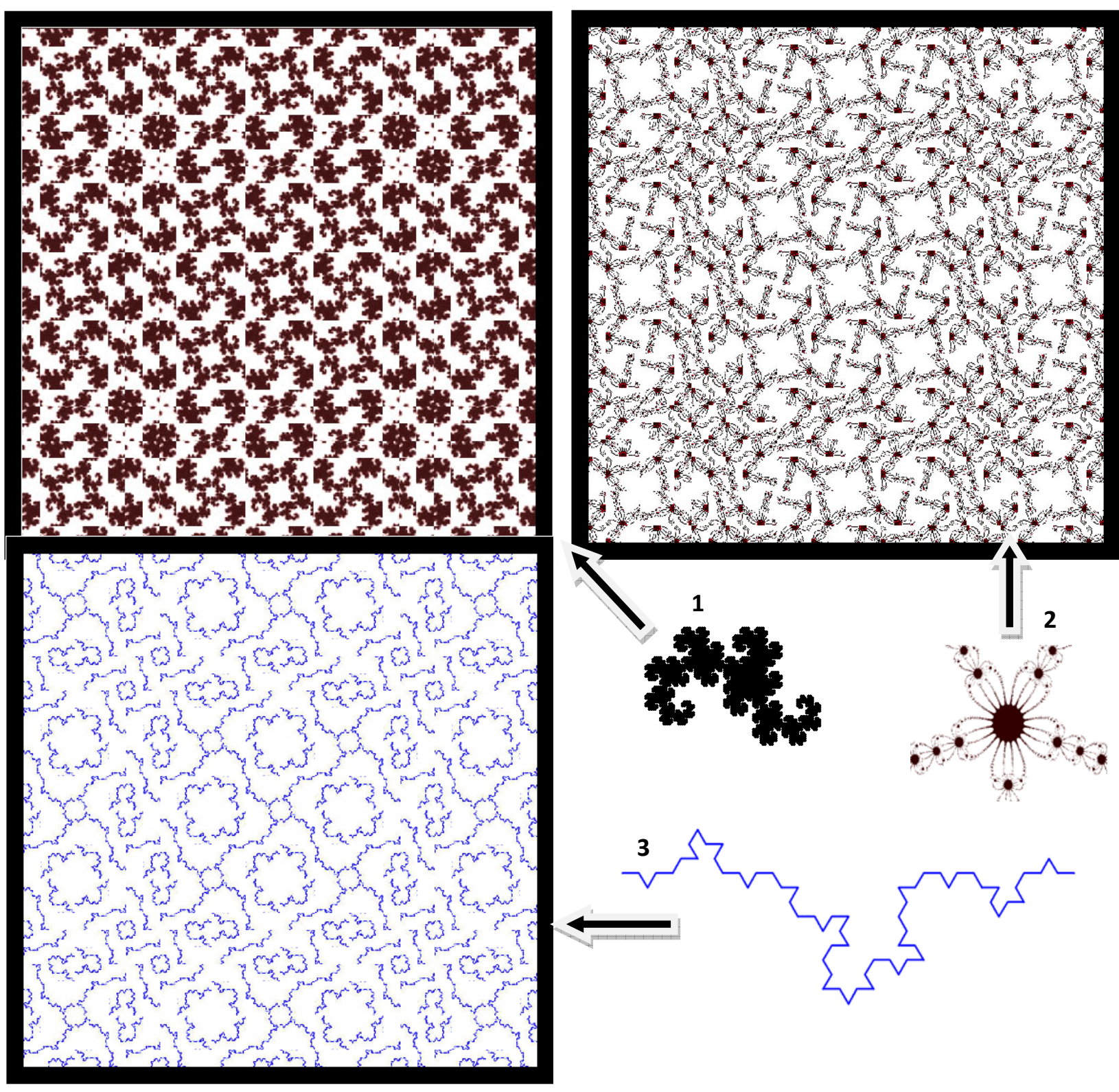

Figure 8.

Thue-Morse L-System tiles the Dragon Curve (1), Newton's method (2), and random Koch's Snowflake (3). 
Thus, in two dimensional form, if we have the first generation written as

Gen_1:=[ $\left.\begin{array}{ll}0 & 1 \\ 1 & 0\end{array}\right]$

then the second is,

Gen_2 $_{-}:=\left[\begin{array}{cccc}0 & 1 & 1 & 0 \\ 1 & 0 & 0 & 1 \\ 1 & 0 & 0 & 1 \\ 0 & 1 & 1 & 0\end{array}\right]$

Thus, the processes of the tiling would be as shown in figure 9.
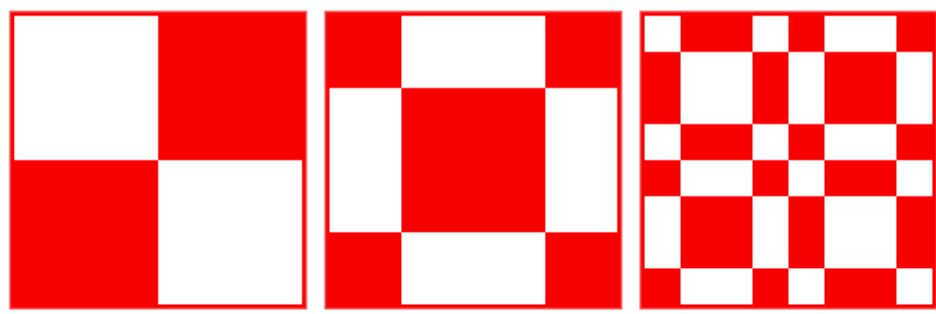

Figure 9.

The generations of 2 dimensional Thue-Morse L-System Tiling.

Some fractal collections other than previously shown Mandelbrot and Julia sets could also be incorporated to enrich the batik ornamentation. Figure 8 shows the three of the most famous one. The first is the Newton's method as elaborated in detail by Peitgen \& Saupe (1988). The basic pattern become the motif that is tiled by using the Thue-Morse algorithm. It is interesting to find the emerged big picture of the yielded ornaments. The second pattern shown in the figure is based on the dragon curve. The elaboration of this method can be seen in Wells (1991: 59). The dragon curve is basically made up as recursive nonintersecting curve, but for the need as the basic motif here, we fill the coverage of the curve in brown color as presented in the figure. The last example is the modification to the Koch's Curve, inspired by the algorithm of the famous Koch's Snowflake (Wells, 1991: 135-6). Originally the Koch's Snowflake is a closed curve or just slightly made with a single line as initial condition. However, in the model we use here, we made random angle for the growing iterative lines in our algorithm as shown in the figure. Apparently, from the figure we could clearly see another emerging ornamentation that is applicable for batik decorations by using transformation that can simply be written as,

\section{fractal $\stackrel{\text { Thue-Morse L-System Tiling }}{\longrightarrow}$ batik / isen}

However, this transformation is still can be categorized as the first mode of the batik fractal. Furthermore, we could use this kind of transformation to create the second mode of the batik hybrid by making the superposition of traditional batik designs and the tiling method as the isen of the batik. An exemplification is shown in figure 11, where we extract a motif originally recognized as part 
of the wastra bangbangan tiled with the Peano Curve which is tiled with the Thue-Morse algorithm. Peano Curve in its nature is a kind of space-filling algorithm (cf. Weisstein, 2008) and can be regarded also as a Lindenmayer system (Peitgen \& Saupe, 1988), one of the most famous fractal generating mechanisms. Here, instead of letting Peano curve fills all the landscape, we implement the Thue-Morse tiling system to fill the empty spaces of the motif taken from the traditional bangbangan batik.

Furthermore, the method of hybrid batik does not always placing the generated fractal to becoming the isen of the batik. Both can also become the major dominant pattern of batik. An interesting pattern possible actualization is presented in figure 12. It is made of randomized Sierpinsky triangle mixed with the traditional parang motif.

The Sierpinski triangle is one of the oldest and mostly discussed generated fractal geometrical shape (for detail see Wolfram, 2002 and Peitgen \& Saupe, 1988). However, the triangle we show here is the modified one by incorporating randomness in the generating processes. This becomes an interesting aesthetics since it is mixed with the very regular parang batik motif. The two motifs are dominant, not one of them become the klowong or isen but expected to emerge a harmonious things in its design. Figure 11 and 12 are different in the generating of batik ornamentation but both can be categorized as the same: the hybrid batik, where communication of computationally generative traditions meets with the manual counterparts.

3) Innovative Batik: The implementation of the random image tessellation and/or tiling algorithm from original batik ornaments as the isen of the traditionally recognized (batik) patterns.

The aesthetic horizon is widespread in the infinity and a lot basic motif can be incorporated in the generative batik ornamentation. This is actually become a particular challenge in the notion of generative batik ornamentation since we are bounded to the rule of what we call batik in eq. 1 and figure 3 on what we can call mbatik and batik. Batik is somehow the process in its wholeness from the preparing the materials to the appreciation.

The batik innovation used traditional based motif (which is not necessarily the shape is originally use as common batik ornaments) following the steps of the process of the ornamentation to result a new kind of batik. Here, the creativity on choosing the aesthetically correct basic motif, be it klowong or isen, is left to the artist. Computational steps could however only capture the pseudoalgorithmic steps of batik.

Simply saying, the innovation batik used traditionally recognized motif to becoming the source of the ornamentation. The algorithm used to create the decoration, be it tiling method or merely incorporated the well known motif as a grand design of the batik, can be anything. Thus, the role of the computational processes is just the emergence of the resulting big picture patterns. As an example is shown in figure 14. The basic motif used is the one commonly used in Jambinese batik, the melati motif. The basic small motif is then used to create the innovative batik by using the LSystem Thue-Morse algorithm and interestingly a new pattern is emerged. 


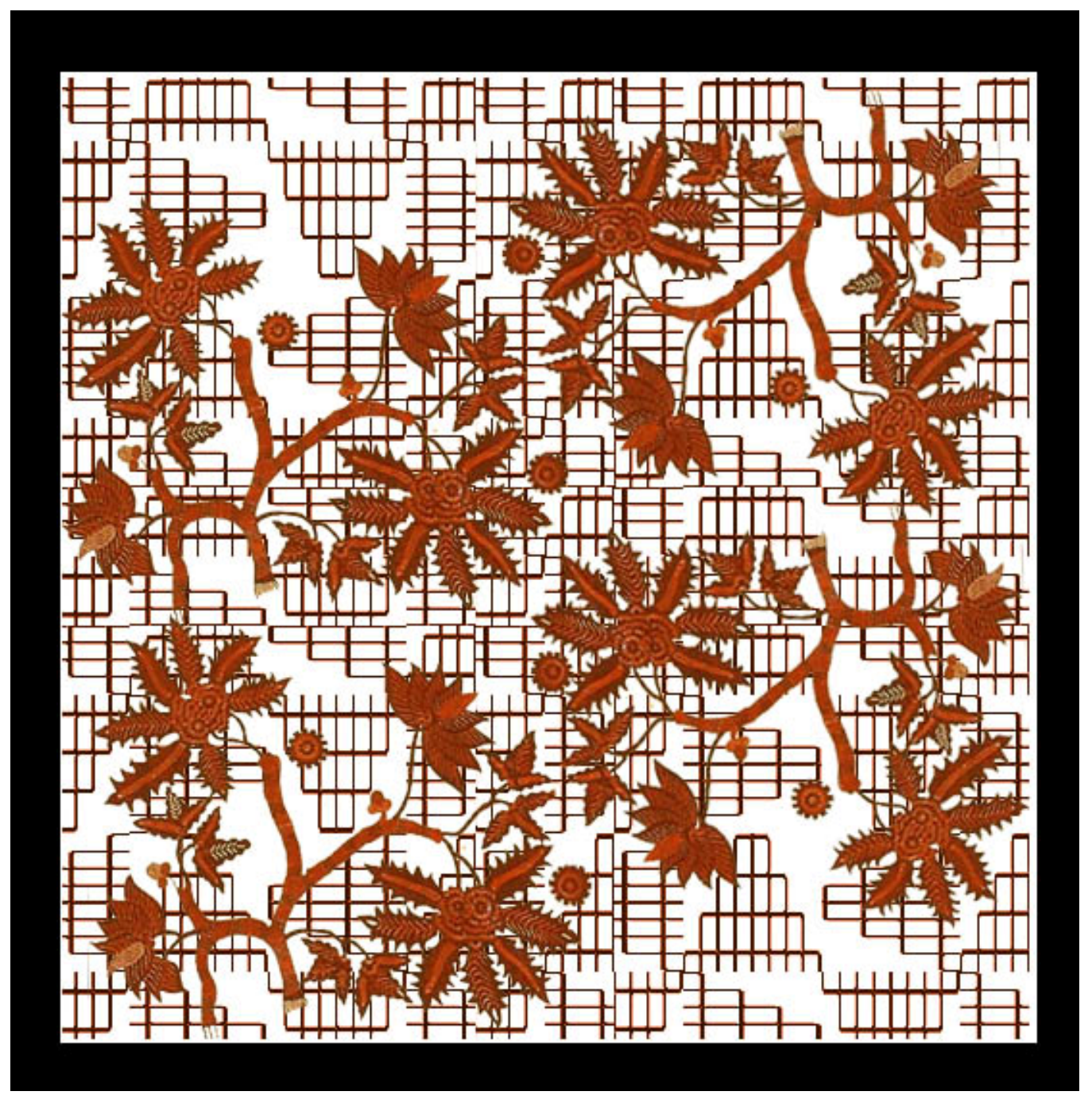

Figure 11.

The generated Peano Curve tiled with Thue-Morse algorithm used as the filling to the traditional batik ornament originally known as part of the stamped batik (batik cap) of wastra bangbangan.

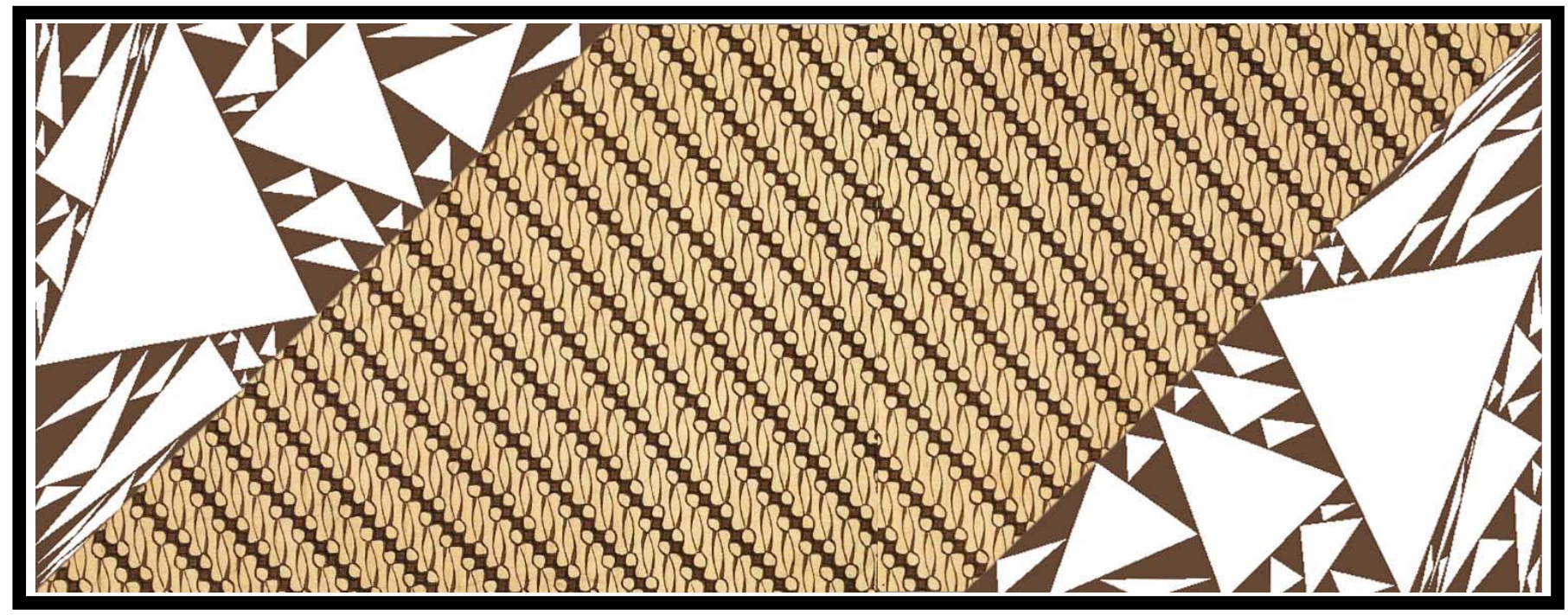

Figure 12.

The design that can be actualized as carrying cloth with parang motif, originally recognized as slarak kendang for its sacred musical verses in the right and left side but now replaced with the random sierpinski triangle. 


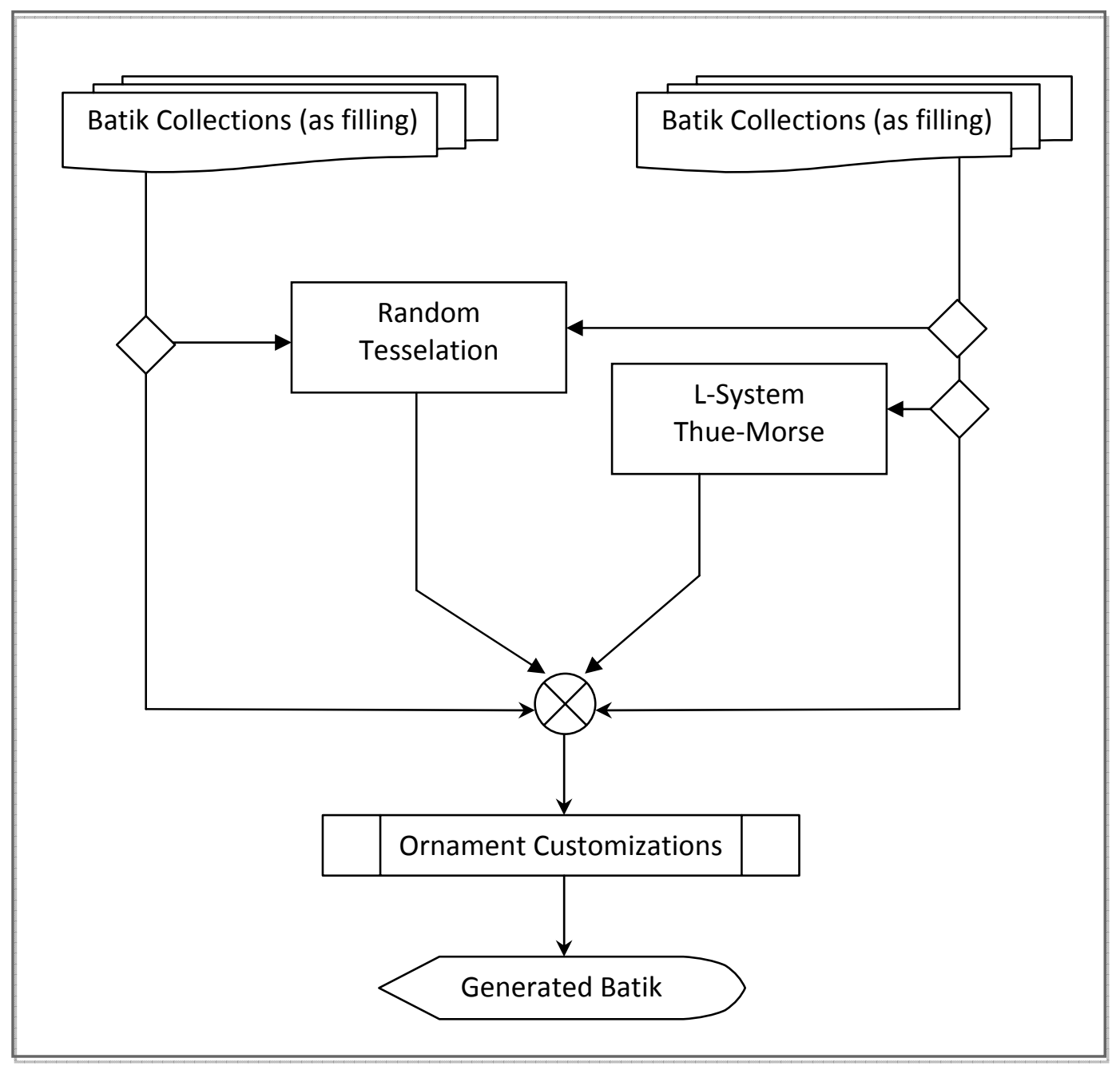

Figure 13

Batik Innovation.

Another interesting implementation of this kind of innovative batik is by employing what we have outlined previously as depicted in the left side in figure 2: the random tessellating processes. By using this kind of method, we could possibly "superpose" a lot of basic traditional motif into a textile space to be processed. As an exemplification is shown in figure 15. In the figure, the random-sized tessellation is applied to superpose three main motif from the same name but different variations of traditional Javanese sawat. The emerging big picture is interesting and could be enjoyed as "typical" batik ornamentation. 


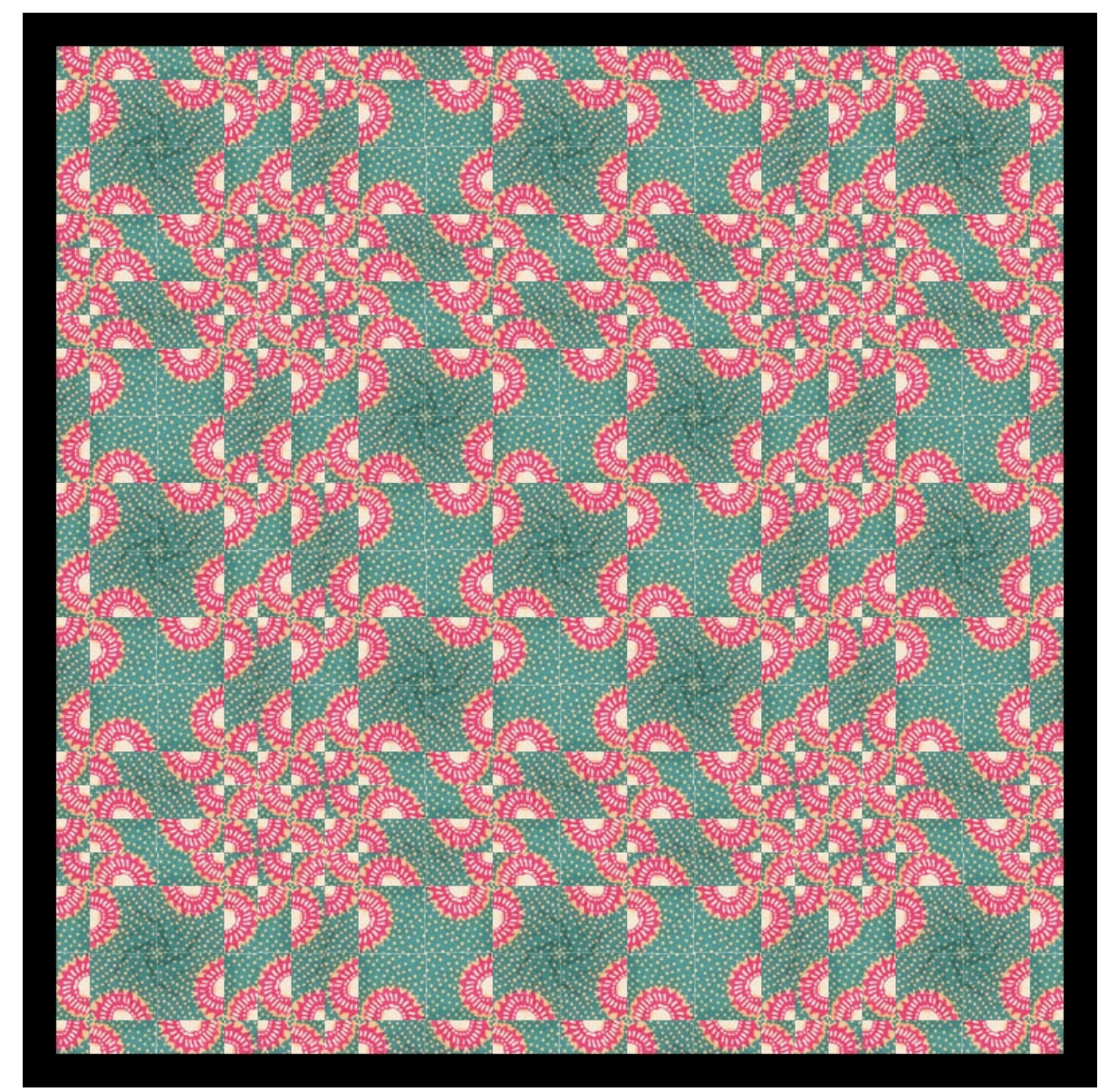

Figure 14

The Melati, one motif from Jambinese batik tiled with L-System Thue-Morse algorithm.

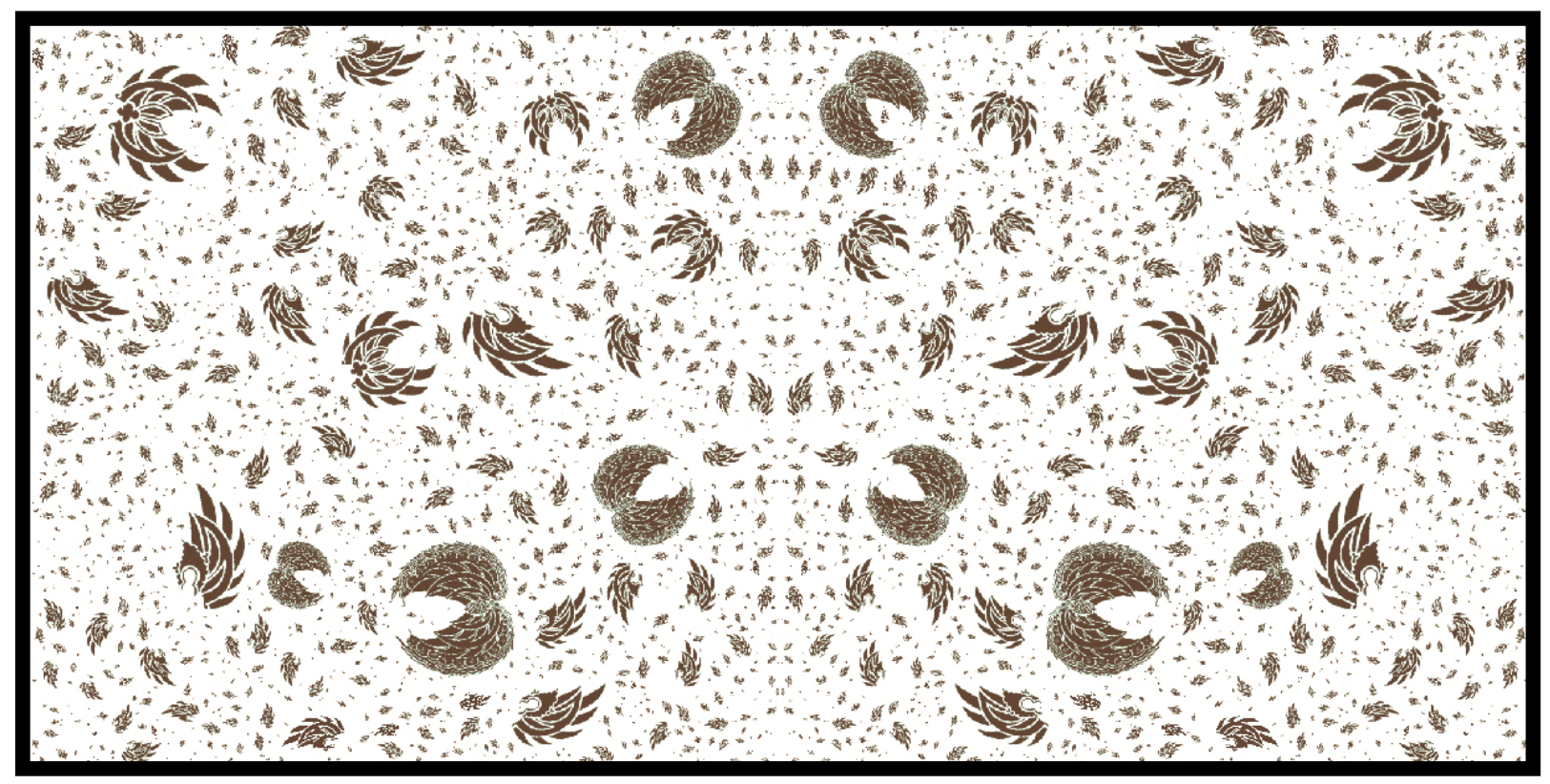

Figure 15

Three variation of commonly used basic motif recognized as sawat superposed in the random tessellations algorithms as innovative batik. 


\section{Concluding Remarks}

We discussed things behind the terminology of batik as one of worldly recognized Indonesianspecific textile products. In addition to the complex patterns emanated aesthetically from a batik crafting, batik cannot be reduced in certain aspects of its properties. Formally speaking, the properties of batik should be seen as a whole process from the decision on its material, the mbatik processes including the ornamentation, and even to the appreciation on how people traditionally used batik. These properties, interestingly do not emphasize batik on the ornamentation but the batik crafting processes. The pseudo-algorithmic of mbatik is then showed might exploited the computational procedures capturing the self-similarity showed in batik ornamentation.

The computationally generative batik can be conducted in three categories, i.e.: using mathematical fractal geometry with certain image customization, e.g.: the coloring techniques, zooming and changing variables of the computationally yielded fractal images. This method is related to the selfsimilarity properties of batik ornamentation. The second way is labeled as hybrid batik, in which using the fractal shapes as well as the traditional batik basic shapes, be it as the main designs or the harmonization or isen. The third is the innovative batik, in which the processes include wide ranging main motifs as basic pattern used in the generative process. It is worth noting, that interestingly, the sources of the fundamental motif in the process are not necessarily a widely known basic batik pattern. It is also discussed that the computational generative batik employs one or possibly both the L-system Thue-Morse algorithm for the need of image tiling and the image random tessellating processes.

Here we have showed that the computation can be exploited to enhance and enrich the innovations related to the "standard" traditional heritage. Even though the computation will never be able to replace the process of batik crafting as well as, but most importance, the corresponding aesthetical aspects, the computationally generative batik ornaments has shown us that the meeting of traditional crafting and modern computation could lead us to the unlimited creativity. A thing that is so much demanded by our nowadays-economic system.

\section{Acknowledgement}

I thank Yun Hariadi for discussions, Alga Indria for some literatures and discussions related to visual art, Arif Heryanto for his survey results for Jambinese batik artists, and colleagues in Indonesian Archipelago Culture Initiatives (IACI) for some important data and inputs.

\section{References}

Bak, P.(1996). How Nature Works: The Science of Self-organized Criticality. Springer-Verlag.

Barnsley. M. F. (1988). Fractals Everywhere. Academic Press.

Doellah, H. S. (2002). Batik: The Impact of Time and Environment. Danar Hadi.

Hariadi, Y. (2008). “Fractal Geometry on Batik". Journal of Social Complexity 3 (2): 41-52.

Hasanudin. (2001). Batik Pesisiran: Melacak Pengaruh Etos Dagang Santri pada Ragam Hias Batik. Kiblat. 
Kappraff, J. (2002). Beyond Measure: A Guided Tour Through Nature, Myth, and Number. World Scientific.

Kerlogue, F. (1997). Scattered Flowers: Textiles from Jambi, Sumatera. Center for South-East Asian Studies. University of Hull.

Malkevitch, J. (2003). "Mathematics and Art". Feature Column April 2003. American Mathematical Society. URL: http://www.ams.org/featurecolumn/archive/art1.html

Mandelbrot, B. (1982). The Fractal Geometry of Nature. W. H. Freeman.

Peitgen, H-O., \& Saupe, D. (1988). The Science of Fractal Images. Springer-Verlag.

Situngkir, H. (2005). "What is the Relatedness of Mathematics and Art and Why We Should Care?". BFI Working Paper Series WPK2005.

Sondari, K. \& Yusmawati. (2000). Batik Pesisir. Cultural Media Development Project - Departement of Education and Culture, Republic of Indonesia.

Weisstein, E. W. (1999). MathWorld-A Wolfram Web Resource. URL:

http://mathworld.wolfram.com/

Wolfram, S. (2002). A New Kind of Science. Wolfram Media Inc.

Wells, D. (1991). The Penguin Dictionary of Curious and Interesting Geometry. Penguin

Weisstein, E. W. (2008). "Peano Curve." MathWorld--A Wolfram Web Resource. URL:

http://mathworld.wolfram.com/PeanoCurve.html 